Laser Chem., 1999, Vol. 19, pp. 287-289

Reprints available directly from the publisher Photocopying permitted by license only
(C) 1999 OPA (Overseas Publishers Association) N.V. Published by license under the Harwood Academic Publishers imprint, part of The Gordon and Breach Publishing Group.

\title{
DETERMINATION OF THE LOWEST EXCITED STATE OF METAL COMPLEXES OF DIPYRIDO[3, 2-a:2', 3'-c]PHENAZINE
}

\author{
MARK R. WATERLAND* and KEITH C. GORDON \\ Chemistry Department, University of Otago, PO Box 56, Dunedin, \\ New Zealand
}

(Received 6 April 1997)

The nature of the lowest excited state of rhenium and copper complexes of dipyrido[3,2a: $2^{\prime}, 3^{\prime}$-c]phenazine (Dppz) has been determined using Resonance Raman, TimeResolved Resonance Raman Spectroscopy and Spectroelectrochemistry. Comparison of spectroelectrochemical data and excited state data show that for the complexes studied no reduced ligand bands are observed in the excited state spectra thus the lowest excited states are all Ligand Centred in nature. The use of substituents at the 11 and/or 12 position of the ligand has no effect on the excited state ordering.

Keywords: Dipyridophenazine; lowest excited state; Time-Resolved Resonance Raman; Resonance Raman; spectroelectrochemistry

In recent years there has been much interest in solar energy conversion schemes based on metal polypyridyl complexes [1]. In these schemes the polypyridyl complexes sensitize the formation of a chargeseparated state via population of a low energy Metal-to-Ligand Charge-Transfer (MLCT) excited state. An effective sensitizer requires a lowest excited state with MLCT character with a long lifetime, a high oscillator strength in the solar spectrum and photophysics which efficiently populate the lowest excited MLCT state. In the MLCT excited state an electron is localised on a $\pi^{*}$ orbital of the ligand and so the ligand is formally reduced whereas a LC excited state is

\footnotetext{
* Corresponding author.
} 
formally a $\pi \pi^{*}$ excited state. Metal complexes of the Dppz ligand $\left(\mathrm{dppz}=\right.$ dipyrido[3,2-a:2 $2^{\prime}, 3^{\prime}$-c]phenazine $)$ show promise as sensitizers because of their long excited state lifetimes. However, the Dppz ligand has a low lying $\pi \pi^{*}$ excited state which when complexed with $\mathrm{Cu}(\mathrm{I})\left(\mathrm{PPh}_{3}\right)_{2}{ }^{+}$or $\operatorname{Re}(\mathrm{I})(\mathrm{CO})_{3} \mathrm{Cl}$ moieties can be the lowest excited state. This study determines the nature of the lowest excited states of $\mathrm{C} 1(\mathrm{CO})_{3} \operatorname{Re}(\mathrm{I}) \mathrm{L}$ and $\left(\mathrm{PPh}_{3}\right)_{2} \mathrm{Cu}(\mathrm{I}) \mathrm{L} \quad \mathrm{BF}_{4}$ complexes where $\mathrm{L}$ is the Dppz ligand with benzo, methyl and methoxy groups at the 11 and/or 12 positions. The effect of the substituents on the excited state ordering is also investigated.

To assign the nature of the lowest excited state, a combination of Resonance Raman (RR) Spectroscopy, Time-Resolved Resonance Raman $\left(\mathrm{TR}^{3}\right)$ Spectroscopy and Spectroelectrochemistry was used. The RR scattering was generated using $457.9 \mathrm{~nm}$ excitation, collected with a backscattering geometry and imaged onto a LN-CCD using a single-stage spectrograph. The pulsed $448.2 \mathrm{~nm}$ excitation for the $\mathrm{TR}^{3}$ experiments was generated by Stimulated Raman Scattering through Acetonitrile using the frequency-tripled output from a Q-Switched Nd: YAG laser ( $10 \mathrm{~ns}$ pulse width). The various Raman shifted wavelengths were separated using a Pellin-Broca prism. The Raman Spectra of the radical anions of the ligands were produced using either an Optically Transparent Thin Layer Electrode cell or by imaging the scattering from a reduced species bound to the surface of a silver electrode.

The $457.9 \mathrm{~nm}$ spectra show features attributable to ligand modes at $1590(\mathrm{w}), 1450(\mathrm{~m}), 1410(\mathrm{~s}), 1320(\mathrm{~s})$ and $1180(\mathrm{w}) \mathrm{cm}^{-1}$. These features are assigned as ground state features. The $448.2 \mathrm{~nm}$ spectra show fewer features most notably at $1450(\mathrm{w}), 1400(\mathrm{~s})$ and $1280(\mathrm{~m}) \mathrm{cm}^{-1}$. These features are assigned as excited state features on the basis of comparison with the ground state spectra. Each of the new excited state features increase in intensity with an increase in laser fluence relative to solvent features which adds further support for this assignment. The creation of a significant excited state population is consistent with the long lifetimes (>100 ns) determined using emission decay [2]. There are only two strong features observed in the reduced species spectra, at 1590 and $1360 \mathrm{~cm}^{-1}$. Note of the features observed in the reduced species are observed in the excited state data thus the excited state is LC in nature. Electron withdrawing and donating groups on the 
ligand have no effect on the excited state ordering as the data for all the complexes studied show excited state features which aren't present in the reduced species spectra.

\section{References}

[1] Kalyanasundaram, K. (1982). Co-ord. Chem. Rev., 46, 159-244.

[2] Waterland, M. R. and Gordon, K. C., results to be published. 